\title{
Optical necklaces generated by the diffraction on a stack of dielectric wedges
}

\author{
Yana Izdebskaya ${ }^{\mathrm{a}, \mathrm{b}, *}$ \\ ${ }^{a}$ Nonlinear Physics Centre, Research School of Physical Sciences and Engineering, Australian National University, Canberra ACT 0200, Australia \\ ${ }^{\mathrm{b}}$ Department of Physics, V.I. Vernandsky Taurida National University, Simferopol 95007, Crimea, Ukraine \\ Received 8 February 2008; accepted 11 February 2008 \\ Available online 4 March 2008 \\ Communicated by V.M. Agranovich
}

\begin{abstract}
We demonstrate that the regular ring-shaped arrays of Gaussian beams, or optical necklaces, can be generated using diffraction on a stack of dielectric wedges. A condition for self-similarity and structural stability of the beams has been derived and shows good comparison with experimental data.
\end{abstract}

(C) 2008 Elsevier B.V. All rights reserved.

PACS: 41.85.Ja; 41.85.Ct

Keywords: Singular beams; Optical wedge

\section{Introduction}

During recent decade an increasing attention has been given to the problems of the formation and transport of optical vortices in linear [1,2] and nonlinear [3,4] optical media. Optical vortices appear in coherent fields as the isolated points of total destructive interference with amplitude vanishing and phase undefined or singular [1]. A challenging task here is to achieve structural stability of complex beams, in particular the rotating beams carrying multiple phase singularities. Theoretical analysis of these so-called "spiraling" beams was developed for linear media (free propagation) [5] and the condition of their structural stability was recently derived [6]. In nonlinear media, however, most of the studies were focused on one particular class of spiralling beams, namely a ring-like array of simple bell-shaped (Gaussian) beams in the form of optical necklaces [7], soliton clusters [8], and azimuthons [9]. Thus, the problem of efficient experimental generation of structurally stable optical necklaces remains par-

\footnotetext{
* Correspondence address: Nonlinear Physics Centre, Research School of Physical Sciences and Engineering, Australian National University, Canberra ACT 0200, Australia.

E-mail address: yvi124@rsphysse.anu.edu.au.
}

ticularly important for applications in nonlinear singular optics.

The straightforward way to construct optical necklaces is to consider superposition of the off-axis Gaussian beams arranged into a ring-shaped array and tilted along the generatrix of the hyperboloid of rotation $[6,10]$, similar to the cluster of fundamental solitons in nonlinear media [8]. In this approach each beam in the array must be aligned precisely as the small deviations from self-similar shape of the whole cluster may lead to unstable propagation. Therefore, for large number of beams ("pearls" in the necklace), the method is not efficient. Another approach is to use holographic methods $[11,12]$, such as computer-generated holograms on the spatial light modulator. However, with this approach it is difficult to control simultaneously the spatial modulation of the amplitude and phase and again only relatively simple (lowest order) optical necklaces can be generated efficiently.

In this Letter we demonstrate, both theoretically and experimentally, that the diffraction on the stack of optical wedges can be tailored to generate structurally stable optical necklaces equivalent to the arrays of Gaussian beams carrying optical vortices. Moreover, by changing the stack parameters it is possible to achieve fine tuning of the necklace profile. In the rest of the Letter we revisit the problem of the diffraction on a single 


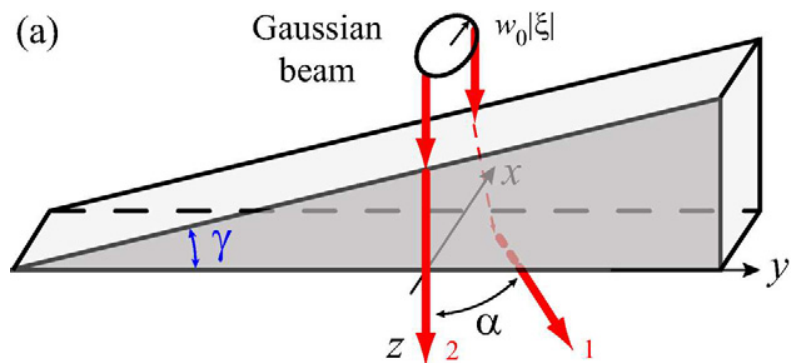

(b)

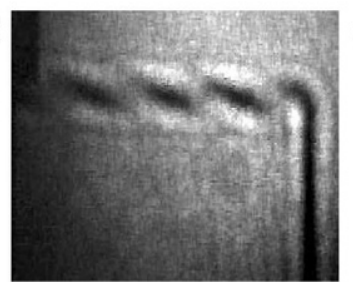

$w_{0}=5 \mathrm{~mm}$

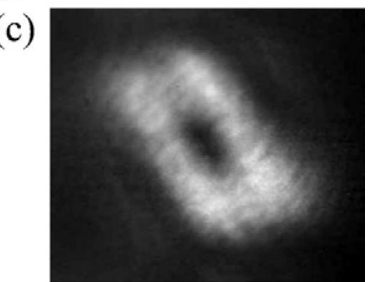

$w_{0}=1 \mathrm{~mm}$

Fig. 1. (a) Sketch of a Gaussian beam diffracting on the edge of a single optical wedge with $\gamma=9 \times 10^{-4}$. (b), (c) Experimental results of the diffraction of two different beams [13], with the waist parameter equal (b) $w_{0}=5 \mathrm{~mm}$ (chain of vortices) and (c) $w_{0}=1 \mathrm{~mm}$ (single vortex).

dielectric wedge and on the stack of wedges, obtain the target structurally stable profile of the array of Gaussian beams, and derive a simple condition allowing to optimize the diffraction pattern and fulfill stability requirements. In the last section before concluding the Letter we compare theoretical and experimental data for the optical necklaces with four, six, and eight pearls.

\section{Diffraction on a wedge}

First we recall the results of Refs. [13-15] on the diffraction on a single dielectric wedge. We assume that a Gaussian beam with the envelope $\Psi_{0}(x, y, z)=\frac{1}{\xi} \exp \left(-\frac{x^{2}+y^{2}}{w_{0}^{2} \xi}-i k z\right)$ is passing through the optical wedge as shown in Fig. 1. Here $w_{0}$ is the beam waist in the plane $z=0, \xi=1-i z / z_{0}, z_{0}=k w_{0}^{2} / 2$ is the Rayleigh length, $k=2 \pi / \lambda$ is the wave number and $\lambda$ is the wavelength of incident laser beam. The wave function of the beam passed through the wedge can be calculated using Kirchhoff diffraction integral

$$
\begin{aligned}
\Psi_{1}(x, y, z)= & \Psi_{0}(x, y, z) \\
& \times\left\{\exp \left(i k\left(n_{w}-1\right)(y \gamma / \xi+h)\right) \operatorname{erfc}(X)\right. \\
& +\operatorname{erfc}(-X)\}
\end{aligned}
$$

here $X=x \sqrt{i k / 2 z \xi}$ and the wedge is characterized by the vertex angle $\gamma$, the refractive index $n_{w}$, and the thickness $h$ in the plane $y=0$.

The process of vortex formation can be explained if we consider two beams 1 and 2 in Fig. 1(a), one is passing through the wedge and another propagating in free space. Optical vortex will be formed when two beams will interfere destructively, i.e., when the phase difference between both waves is

$\theta_{m}=(2 m+1) \pi, \quad m=1,2, \ldots$

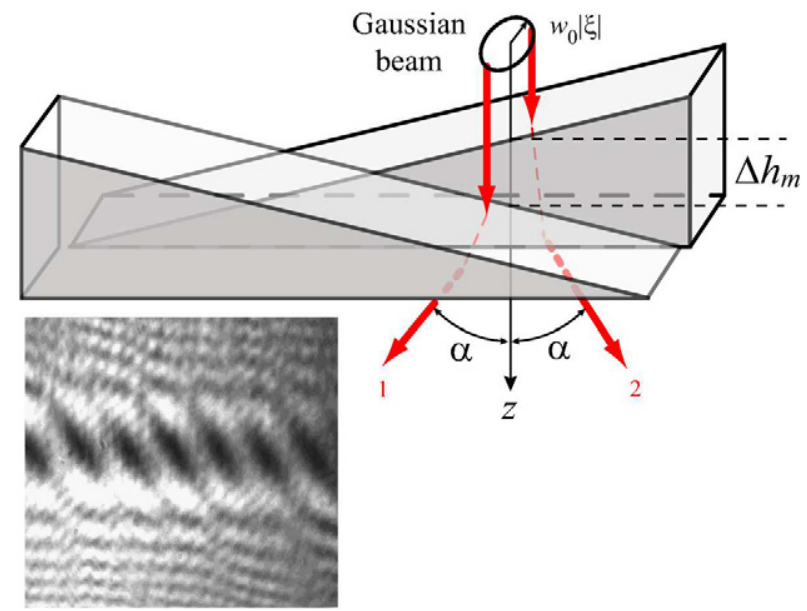

Fig. 2. The sketch of a symmetric double-wedge system and the diffraction pattern from a broad Gaussian beam.

In other words, the optical path difference $h\left(n_{w}-1\right)=\theta_{m} / k$, entering Eq. (1), must equal half-integer number of wavelengths with the wedge thickness satisfying

$h=h_{m}=\lambda(2 m+1) / 2\left(n_{w}-1\right)$.

It follows that, for sufficiently broad input beam, we obtain chain of vortices enumerated with $m$ (vortex street, see Fig. 1(b)) and positioned in the plane $x=0$ of the wedge-air interface at

$y_{m}=\frac{2 \pi m}{\gamma k\left(n_{w}-1\right)} \sqrt{1+\frac{z^{2}}{z_{0}^{2}}}$.

Tailoring the diffraction means choosing the input beam waist $w_{0}$ appropriately, for example, to isolate a single vortex as in Fig. 1(c), we put the diameter to be equal the distance between two neighboring vortices $2 w_{0}=y_{m}-y_{m-1}$ at $z=0$, i.e., $w_{0}=$ $\pi / \gamma k\left(n_{w}-1\right)$.

The structure of Eq. (1) readily suggests that two terms in the sum are related to the two beams, one passing through the wedge and another in free space, but they enter into the solution non-symmetrically. The asymmetry appears because of the twist of wave front of the refracted beam. Thus the axis of the refracted beam is also inclined in space behind the wedge at an angle

$\alpha=\gamma\left(n_{w}-1\right)$

with respect to the axis of the incident beam.

In the following we will consider a stack of optical wedges and the disadvantage of a single wedge is the lack of an axis of symmetry for two beams after the wedge. Though the tilt angle $\alpha$ is small for thin wedges $\gamma \ll 1$, it will accumulate in the stack of wedges and distort the desired symmetry. To compensate this asymmetry and preserve the initial beam propagation direction $z$, we consider a system of two wedges superimposed as shown in Fig. 2. The second dielectric wedge has the same vertex angle $\gamma$ as the first one but directed in the opposite way, thus the refracted beams in both will have the same tilt in opposite directions. Calculation of the diffraction integral lead to the 
solution similar to Eq. (1) but symmetric with respect to propagation axis $z$

$$
\begin{aligned}
\Psi_{2}(x, y, z)= & \Psi_{0}(x, y, z) \\
& \times\left\{\exp \left(i Y+i \theta_{1}\right) \operatorname{erfc}(X)\right. \\
& \left.+\exp \left(-i Y+i \theta_{2}\right) \operatorname{erfc}(-X)\right\}
\end{aligned}
$$

where $Y=y k \alpha / \xi$ and $\theta_{1,2}=k n_{w} h_{1,2}$ are the phases of the beams passed distances $h_{1,2}$ in the wedge 1 and 2 , respectively. Similar to the previous case of a single wedge, an optical vortex is formed when two beams are out of phase, $\theta_{1}-\theta_{2}=$ $(2 m+1) \pi$, and, therefore, $n_{w} \Delta h_{m}=(2 m+1) \lambda / 2$, here and in Fig. 2 we denote $\Delta h_{m}=h_{1}-h_{2}$.

Eq. (5) represents the superposition of two Gaussian beams propagating at an angle $2 \alpha$ to each other. Calculating the position of vortices (zeros of the field) we obtain $y_{m} / 2$, half the values given by Eq. (3). It means that the chain of vortices in Fig. 2 is similar to Fig. 1(b) but with twice the number of vortices per unit length $[16,17]$. A single vortex from the thin diffracting Gaussian beam has the same profile as in Fig. 1(c) but with the waist parameter twice smaller.

\section{Ring arrays of Gaussian beams}

We aim to employ the condition of the structural stability of the ring arrays of Gaussian beams, obtained in Ref. [6], to the necklace beams generated by the optical wedges. To do that, we recall here the wave function of an array $\Psi_{G}$ of $N$ off-axis Gaussian beams [18] $\Psi_{n}, n=1,2, \ldots, N$,

$\Psi_{G}=\sum_{n=1}^{N} \Psi_{n}=\Psi_{0} \sum_{n=1}^{N} \exp \left(\Phi_{n}\right)$,

where the expression for common Gaussian envelope $\Psi_{0}(r$, $\varphi, z)$ was given above and

$$
\begin{aligned}
\Phi_{n}= & -\frac{r_{0}^{2}+2 r_{0} r \cos \left(\varphi-\varphi_{n}\right)-\alpha^{2} z_{0}^{2}}{w^{2} \xi} \\
& -i \frac{\alpha k r}{\xi} \sin \left(\varphi-\varphi_{n}\right)+i \theta_{n} .
\end{aligned}
$$

Each Gaussian beam is positioned at azimuthal coordinate $\varphi_{n}=$ $2 \pi n / N$ on the ring of radius $r_{0}$ in the waist at $z=0$, and each is assigned a phase value $\theta_{n}=l \varphi_{n}$, here integer $l$ is the topological charge (orbital index) of the ring array. Each beam is tilted by $\alpha$ with respect to the optical axis $z$ so that the beam axes lie on the surface of a hyperboloid of revolution.

The key result derived recently [6] is the condition of the structural stability for ring arrays, distinguishing self-similar beams from the general superpositions in Eqs. (6) and (7)

$r_{0}=\alpha z_{0}$

We apply this constrain to the simplest configuration, which will be analogous to the considered above diffraction on two wedges, namely the case of just two Gaussian beams, $N=2$. It is convenient for the following to present Eq. (6) in explicit form utilizing the coordinate system used above in Eq. (5)

$$
\begin{aligned}
\Psi_{G}(x, y, z)= & \Psi_{0}(x, y, z) \\
& \times\left\{\exp \left(i Y-\alpha k x / \xi+i \theta_{1}\right)\right. \\
& \left.+\exp \left(-i Y+\alpha k x / \xi+i \theta_{2}\right)\right\} .
\end{aligned}
$$

Now we are in a position to apply the stability criteria to the beams diffracted on wedges. We compare Eq. (5) for a pair of wedges with Eq. (9) for a pair of Gaussian beams. First, for a single vortex after the wedges ( $m=0$ in Eq. (2)) the phase difference $\theta_{2}-\theta_{1}=\pi$ in Eq. (5). Similarly, for the unit topological charge $l=1$ of the two-beams array in Eq. (9) we have $\theta_{2}-\theta_{1}=\varphi_{2}-\varphi_{1}=\pi$. Therefore, the only essential difference between two expressions comes with the factors $\operatorname{erfc}( \pm X)$ in Eq. (5) versus the factors $\exp (\mp \alpha k x / \xi)$ in Eq. (9).

Let us analyze these expressions in the far-field $z \gg z_{0}$. We approximate $\xi \simeq-i z / z_{0}$ thus $X \simeq i x k w_{0} / 2 z$ and

$\operatorname{erfc}( \pm X) \simeq 1 \mp i k x w_{0} / \sqrt{\pi} z$

$\exp (\mp \alpha k x / \xi) \simeq 1 \mp i \alpha k x z_{0} / z$.

It is clear that two expressions are equivalent if $w_{0}=\sqrt{\pi} \alpha z_{0}$ so that, finally,

$w_{0}=\frac{\lambda}{\pi^{3 / 2} \gamma\left(n_{w}-1\right)}$.

In short, the diffraction of a Gaussian beam on a pair of optical wedges can be used to generate a structurally stable spiraling pair of Gaussian beams carrying optical vortex if the waist parameter of the incoming beam is given by Eq. (10).

\section{Structurally stable optical necklaces}

The results above can be expanded to a system with an arbitrary number of symmetrically arranged pairs of wedges. Taking into account that each pair, according to Eq. (5), splits the incoming Gaussian beam into two shifted off-axis beams, by superimposing $M$ pairs we can generate optical necklaces with $2 M$ pearls.

As it was done in Ref. [19], we assume that the $m$ th pair of wedges in the vertical stack is twisted around $z$ axis by an angle $\pi m / M, m=1,2, \ldots, M$. Then the superposition of $M$ fields given by Eq. (5) can be represented as

$\Psi_{M}=\Psi_{0} \sum_{n=1}^{2 M} \exp \left(-i \alpha k y_{n}+i \pi n\right) \operatorname{erfc} X_{n}$,

here $\left\{x_{n}, y_{n}\right\}=r\left\{\cos \left(\varphi-\varphi_{n}\right), \sin \left(\varphi-\varphi_{n}\right)\right\}$ with $\varphi_{n}=\pi n / M$ and $X_{n}=x_{n} \sqrt{i k / 2 z \xi}$. On the other hand, the array of $N$ beams in Eqs. (6) and (7), with the condition of structural stability Eq. (8) satisfied, takes the form

$\Psi_{G}=\Psi_{0} \sum_{n=1}^{N} \exp \left(-i \alpha k y_{n}+i l \varphi_{n}\right) \exp \left(-\frac{\alpha k x_{n}}{\xi}\right)$.

It follows that, in the far-field $z \gg z_{0}$ and with Eq. (10) satisfied, the two expressions above describe the same envelope if $l\left(\varphi_{n+1}-\varphi_{n}\right)=\pi$ and we derive

$N=2 M=2 l$, 
Arrays of Gaussian beams

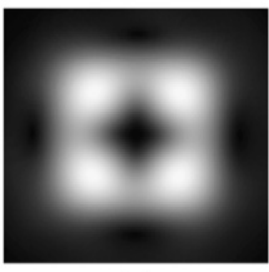

(a)

$N=4, l=2$

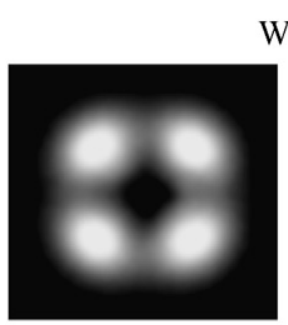

(d)

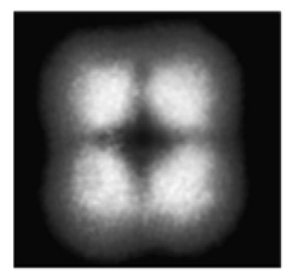

(g)

$M=l=2$

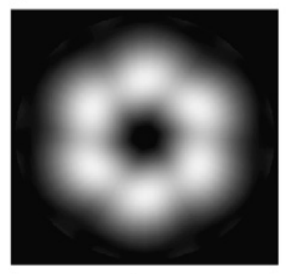

(b)

$$
N=6, l=3
$$

Wedge-generated beams

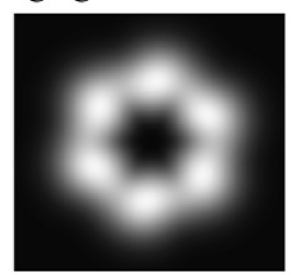

(e)

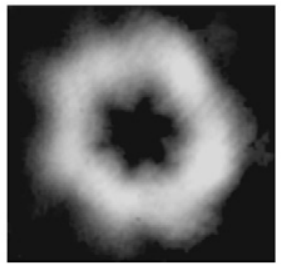

(h)

$M=l=3$

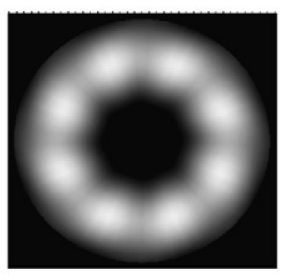

(c)
$N=8, l=4$

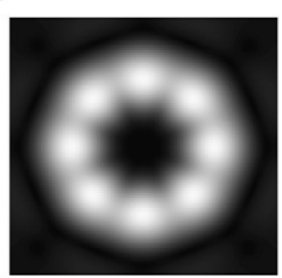

(f)

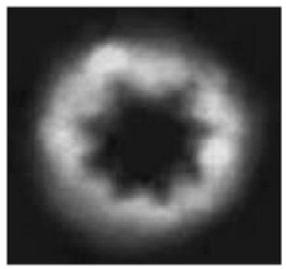

(i)

$M=l=4$
Fig. 3. The intensity distributions of the spiraling optical necklaces calculated (a)-(c) for the superposition of $N=2 l$ Gaussian beams, and calculated (d)-(f) and experimentally recorded (g)-(i) for the diffraction on a stack of $M$ pairs of glass wedges. Parameters are $w_{0}=1.5 \mathrm{~mm}, \lambda=633 \mathrm{~nm}, \gamma=9 \times 10^{-4}$, $n_{w}=1.5$, and $z=5 \mathrm{~m}$.

i.e., the number of pearls in the necklace $N$ is twice the number of pairs of wedges $M$, the later defines the topological charge $l$ of the necklace.

Note that so far we used the limit of the far-field $z \gg z_{0}$ to establish an exact equivalence between fields constructed as the superposition of simple Gaussian beams and those from the diffraction on optical wedges. However, for practical applications it is more convenient to operate fields in the intermediate zone $z \leqslant z_{0}$. Therefore, we compare in Fig. 3 two types of the necklace envelopes for different numbers of topological charge $l$ at the propagation distance $z \simeq 0.45 z_{0}$.

Calculated images of the Gaussian array in Fig. 3(a)-(c) have less pronounced structure of "pearls" than those calculated using Kirchhoff diffraction integral in Fig. 3(d)-(f). This indicates that the phase gradients along the ring between pearls are stronger in the diffraction pattern as should be expected because of the presence of higher harmonics. Still, the differences are subtle and they are definitely below the usual level of deformation of the fields in experiments, cf. Fig. 3(g)-(i). We conclude that the validity of our approximation to the necklace profile can be practically extended from the far to the intermediate field.

We stress here the difference of the optical necklaces carrying optical vortex from the simple superposition of Laguerre-
Gaussian modal beams, such as

$\Psi=\Psi_{0} r^{|l|}\{\exp (i l \varphi)+\exp (-i l \varphi)\} / 2=\Psi_{0} r^{|l|} \cos (l \varphi)$.

The later beam has an intensity distribution very similar to optical necklaces with the same number of intensity maxima along the ring $N=2 l$. However, the simple superposition of two vortex modal beams above, or the multipole beam, is a degenerate case of the optical necklace when all "pearl-beams" in the ring are parallel (i.e., $\alpha=0$ ) and out-of-phase, so that the orbital angular momentum is zero and there is no spatial rotation during propagation. In contrast, varying the parameters of the wedges $\gamma$ and $n_{w}$ we are able to modify the angle $\alpha$ and thus modify phase profile of the necklace. This fine tuning of the necklace opens possibilities to construct different types of azimuthons [9] for the applications in nonlinear singular optics.

\section{Conclusion}

We have analyzed the process of beam diffraction on a stack of dielectric wedges and demonstrated that such a stack can be employed for generation of structurally stable spiraling optical necklaces. Comparison with experimental data in the intermediate diffraction zone shows a good agreement with theoretical results and supports conclusions derived for asymptotes in the far-field.

Our method is limited to the optical necklaces with even number of pearls double the topological charge, and thus it does not cover all the variety of states predicted in nonlinear media. However, its obvious advantage is simplicity and tunability. In addition, optical wedges do not limit operating power as do the spatial light modulators and, therefore, we expect that the method described here will be useful in the ongoing experiments on generation of nonlinear spiraling beams.

\section{Acknowledgements}

The author is grateful to A. Volyar and V. Shvedov for valuable discussions and to A. Desyatnikov for critical reading of the manuscript. This work was supported in part by the Australian Research Council through the Discovery Project.

\section{References}

[1] M. Soskin, M. Vasnetsov, in: E. Wolf (Ed.), Singular Optics, in: Progress in Optics, vol. 42, 2001, pp. 219-276.

[2] M. Vasnetsov, K. Staliunas (Eds.), Optical Vortices, in: Horizons of World Physics, vol. 228, Nova Science, 1999.

[3] C.T. Law, G.A. Swartzlander Jr., Opt. Lett. 18 (1993) 586.

[4] A.S. Desyatnikov, Yu.S. Kivshar, L. Torner, in: E. Wolf (Ed.), Optical Vortices and Vortex Solitons, vol. 47, 2005, pp. 291-391.

[5] E.G. Abramochkin, V.G. Volostnikov, Phys. Usp. 47 (2004) 1177.

[6] Ya. Izdebskaya, V. Shvedov, A. Volyar, J. Opt. Soc. Am. A 25 (2007) 171.

[7] M. Soljacic, M. Segev, Phys. Rev. Lett. 86 (2001) 420.

[8] A.S. Desyatnikov, Yu.S. Kivshar, Phys. Rev. Lett. 88 (2002) 053901.

[9] A.S. Desyatnikov, A.A. Sukhorukov, Yu.S. Kivshar, Phys. Rev. Lett. 95 (2005) 203904.

[10] Ya. Izdebskaya, T. Fadeyeva, V. Shvedov, A. Volyar, Opt. Lett. 31 (2006) 2523.

[11] I. Basistiy, V. Bazhenov, M. Soskin, M. Vasnetsov, Opt. Commun. 103 (1993) 422 
[12] H. He, N.R. Heckenberg, H. Rubinsztein-Dunlop, J. Mod. Opt. 42 (1993) 217.

[13] V. Shvedov, Ya. Izdebskaya, A. Alexeev, A. Volyar, Techn. Phys. Lett. 28 (2002) 256.

[14] Ya. Izdebskaya, V. Shvedov, A. Volyar, Ukr. J. Phys. Opt. 5 (2004) 96.

[15] X.-C. Yuan, B.P.S. Ahluwalia, S.H. Tao, W.C. Cheong, L.-S. Zhang, J. Lin, J. Bu, R.E. Burge, Appl. Phys. B 86 (2007) 209.
[16] Ya.V. Izdebskaya, V.G. Shvedov, A.V. Volyar, Proc. SPIE 6023 (2004) 6023OT.

[17] J. Lin, X.-C. Yuan, J. Bu, H.L. Chen, Y.Y. Sun, R.E. Burge, Opt. Lett. 32 (2007) 2170.

[18] A. Volyar, V. Shvedov, Ya. Izdebskaya, T. Fadeyeva, A. Rubass, Ukr. J. Phys. Opt. 7 (2006) 79.

[19] Ya. Izdebskaya, V. Shvedov, A. Volyar, Opt. Lett. 30 (2005) 2472. 